\title{
A prospective trial evaluating the role of mesothelin in undiagnosed pleural effusions
}

\author{
Clare E. Hooper*," Anna J. Morley*, Paul Virgo`, John E. Harvey", \\ Brennan Kahan ${ }^{+}$and Nick A. Maskell ${ }^{\#}$
}

ABSTRACT: Mesothelin has been proposed as a useful tool in the diagnosis of malignant pleural mesothelioma (MPM). We aimed to examine its diagnostic utility and the impact of renal impairment on results.

We prospectively recruited 230 patients with new undiagnosed pleural effusions, testing serum $(n=216)$ and pleural fluid $(n=206)$ mesothelin (by ELISA) during the initial consultation.

$28(12 \%)$ out of 230 patients had MPM. Serum mesothelin gave sensitivity $59.3 \%$, specificity $64.7 \%$, negative predictive value (NPV) $91.2 \%$, positive predictive value (PPV) $20.5 \%$, and pleural fluid sensitivity $\mathbf{7 2 . 0} \%$, specificity $\mathbf{8 7 . 5} \%$, NPV $95.5 \%$, PPV $46.2 \%$ for distinguishing effusions due to MPM. In a matched comparison, diagnostic characteristics of pleural fluid mesothelin were superior to serum $(p=0.0001)$. Serum mesothelin levels in patients without MPM were higher in patients with renal impairment $(p=0.007)$ while pleural fluid levels were unaffected. $19(54 \%)$ out of 35 patients with a benign pleural effusion and an estimated glomerular filtration rate $\leqslant 59 \mathrm{~mL} \cdot \mathrm{min}^{-1}$ had a false-positive serum mesothelin result.

The diagnostic accuracy of pleural fluid mesothelin is superior to that of serum and is unaffected by renal function. In patients with a low pre-test probability of mesothelioma, a negative mesothelin test could be reassuring, because of its high NPV. Routine use of mesothelin testing in undiagnosed pleural effusions at presentation appears to be unhelpful.

KEYWORDS: Benign asbestos-related effusion, biomarker, diagnosis, malignancy, mesothelioma, predictive value

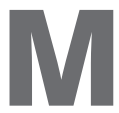
alignant pleural mesothelioma (MPM) is an increasingly common malignancy worldwide with a predicted peak in incidence between 2015 and 2025 in many countries [1]. Most patients present with a pleural effusion and associated breathlessness, a clinical picture with a wide range of possible malignant and benign aetiologies [2]. The diagnosis of MPM can be elusive, particularly in frail patients who are unfit for the most invasive of available diagnostic procedures such as video-assisted thoracoscopy and biopsy [3]. Pleural fluid cytology is of low sensitivity and discrimination between mesothelial cellular atypia due to inflammation and MPM can be challenging [4]. Computed tomography (CT) scan appearances, while of reasonable specificity for identifying malignant thickening of the pleura, distinguish poorly between MPM and adenocarcinoma [5].
A history of asbestos exposure is common amongst patients presenting with a new pleural effusion, many of whom are ultimately diagnosed with benign disease but must be investigated extensively to exclude a malignant diagnosis [3]. In particular, the diagnosis of benign asbestosrelated pleural effusion (BAPE) can currently only be made with confidence following benign pleural biopsy and prolonged radiographic follow-up [6]. Serum or pleural fluid biomarkers that could rule out MPM in high-risk groups, accurately raise diagnostic suspicion in lower risk patients and/or clarify non-diagnostic test results would be valuable.

There have been several studies examining the use of serum mesothelin, a 40-kDa glycoprotein product of mesothelial cells, as a diagnostic and/ or screening tool for mesothelioma, and a recent meta-analysis concluded summary estimates of
AFFILIATIONS

*North Bristol Lung Centre,

Southmead Hospital,

\#Academic Respiratory Unit, School

of Clinical Sciences, University of

Bristol, Southmead Hospital,

"Dept of Immunology, Southmead

Hospital, and

+MRC Clinical Trials Unit, Bristol, UK

\section{CORRESPONDENCE}

N.A. Maskell

Academic Respiratory Unit, School of

Clinical Sciences, University of

Bristol, Learning and Research

Building

Southmead Hospital

Bristol BS10 5NB

UK

E-mail: Nick.Maskell@bristol.ac.uk

Received:

Aug 262011

Accepted after revision:

April 022012

First published online:

July 122012 
$64 \%$ sensitivity and $89 \%$ specificity [7] for the test, which can be performed as a commercially available ELISA. Most studies have employed tissue bank samples, selecting defined diagnostic sub-groups rather than true consecutive series of patients presenting in clinical practice.

The two largest studies to date, examining the use of pleural fluid for mesothelin testing, gave sensitivities of $67 \%$ and $71 \%$ and specificities of $98 \%$ and $89 \%$ for the diagnosis of mesothelioma [8, 9]. DAVIES et al. [9] demonstrated promising diagnostic characteristics for pleural fluid mesothelin in the clarification of nondiagnostic pleural fluid cytology.

Renal impairment has recently been shown to be an independent predictor of elevated serum mesothelin in patients without pleural disease and false-positive rates of $>50 \%$ have been shown in control populations with stage 3 chronic kidney disease (CKD) or greater $[10,11]$. A further study by HoLLEVOET et al. [12] demonstrated an independent association between age, glomerular filtration rate and body mass index and serum mesothelin. The effect of clinical variables such as renal function on mesothelin levels in pleural fluid has not been fully explored.

The aim of the current study was to apply serum and pleural fluid mesothelin testing to a "real-world" prospective consecutive series of patients presenting with an undiagnosed pleural effusion and examine diagnostic utility throughout the standard investigation pathway, compare accuracy between serum and pleural fluid and establish the impact of renal impairment on results.

\section{MATERIALS AND METHODS}

The study was approved by North Bristol NHS Trust Research Ethics Committee, Bristol, UK (08/H0102/11).

Consecutive patients presenting to the pleural disease service of a large UK teaching hospital between July 2008 and July 2010 with an undiagnosed pleural effusion requiring investigation were approached for consent. All recruited patients gave informed written consent.

All patients underwent a comprehensive clinical assessment adhering to British Thoracic Society Guidelines [6].

Cytology/histology techniques and CT scan reporting protocol are described in the online supplementary material.

\section{Clinical data collection}

Clinical data were collected prospectively throughout the patients' involvement in the study. Case notes were reviewed a further 12 months after trial entry to establish the final diagnosis.

\section{Mesothelin quantification}

Paired serum and pleural fluid samples were collected in serum gel separator tubes and centrifuged at $1,000 \times \mathrm{g}$ for $20 \mathrm{~min}$. The supernatant was stored at $-70^{\circ} \mathrm{C}$ for later assay. Mesothelin concentrations were measured with a commercially available ELISA (Mesomark ${ }_{\mathbb{R}}$; Fujirebio Diagnostics Inc., Malvern, PA, USA). Clinical team members were blind to the mesothelin results.

Method of estimated glomerular filtration rate (eGFR) calculation is given in the online supplementary material.

\section{Final diagnosis and follow-up}

Patients were followed-up to a histological or microbiological diagnosis, to complete resolution of the pleural effusion, to death or for a minimum of 12 months. The final diagnosis of each effusion was established independently by two consultant chest physicians using predefined diagnostic criteria by comprehensive review of investigation results and case notes, blind to the mesothelin result. Patients who did not fulfil any diagnostic category following exhaustive investigation and 12 months follow-up were considered "undiagnosed". Full diagnostic criteria are described in the online supplementary material.

\section{Statistical analysis}

Analyses were performed using STATA (StataCorp, College Station, TX, USA) version 12.0 and Prism (GraphPad Software, La Jolla, CA, USA) version 5.0. Differences in the distribution of serum mesothelin and pleural fluid mesothelin levels were compared between patients with and without MPM. Due to the non-normality of the data (based on the D'Agostino-Pearson omnibus normality test), the Mann-Whitney U-test was used to compare medians.

The diagnostic accuracy of serum and pleural fluid was then compared first using a fixed specificity of 95\% (obtained from a receiver operating characteristic (ROC) analysis), including patients for whom both sample types was available, and secondly, including all available results, using pre-defined cutoff levels that have been previously recommended for use in practice. Serum testing used the test manufacturer's cut-off level of $1.5 \mathrm{nM}$ and pleural fluid testing used the best cut-off established in previous studies of $20 \mathrm{nM}[8,9,13]$. 95\% confidence intervals are given for measures of diagnostic accuracy. Nonparametric ROC curves were obtained, and the area under the curve (AUC) was compared between serum and pleural fluid for the paired data. Patients who were undiagnosed or had "radiographic only" diagnoses of malignancy were excluded from all analysis of diagnostic accuracy.

A sub-group analysis was performed to determine whether significant CKD affected test results. Patients were classified into groups based on eGFR results ( $\leqslant 59 \mathrm{~mL} \cdot \mathrm{min}^{-1}$ (stage $3 \mathrm{CKD}$ or greater) versus $>59 \mathrm{~mL} \cdot \mathrm{min}^{-1}$ ), and the two groups were compared in terms of sensitivity, specificity, positive predictive value (PPV) and negative predictive value (NPV).

\section{RESULTS}

Patient recruitment, inclusions, exclusions and demographics are detailed in figure 1.

Agreement between reviewing consultants with regards to the final diagnosis for cause of effusion was good with an unweighted $\kappa$ concordance score of 0.96 (95\% CI 0.93-0.99).

Table 1 summarises patient diagnoses and mesothelin levels.

\section{Serum and pleural fluid mesothelin levels in diagnostic patient sub-groups}

The median (interquartile range) serum mesothelin level amongst patients with MPM $(n=27)$ was $2.10(0.80-6.91) n M$, significantly greater than in those with confirmed non-MPM diagnosis $(n=176) \quad(1.17 \quad(0.90-1.91) n M ; p=0.04)$. Median 


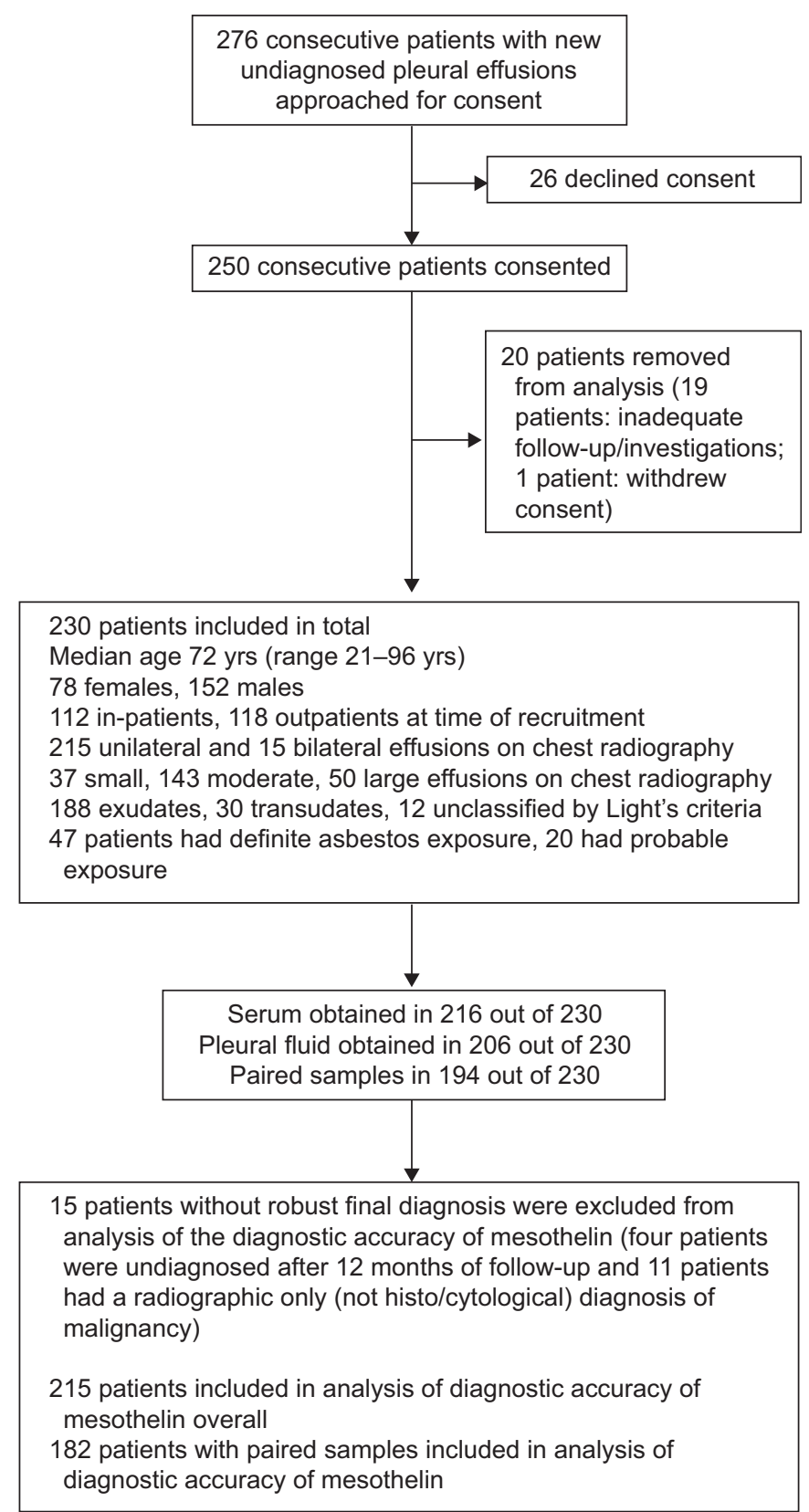

FIGURE 1. Patient recruitment, inclusions, exclusions and demographics.

pleural fluid mesothelin level in patients with MPM $(n=25)$ was $41.2(14.7-70.0) \mathrm{nM}$, also significantly greater than non-MPM patients $(n=168)(5.90(2.85-11.0) n M ; p<0.0001)$.

Positive serum mesothelin results were observed in $26(38 \%)$ out of 69 patients and positive pleural fluid results in $19(26 \%)$ out of 71 patients with non-MPM malignant pleural effusions, including those with adenocarcinoma of unknown primary, breast carcinoma, cholangiocarcinoma, chronic lymphocytic leukaemia, B-cell lymphoma, nonsmall cell lung cancer, ovarian carcinoma, primary peritoneal carcinoma, renal cell carcinoma and small cell lung cancer (fig. 2). A full breakdown of malignant histological sub-types is given in the online supplementary material.

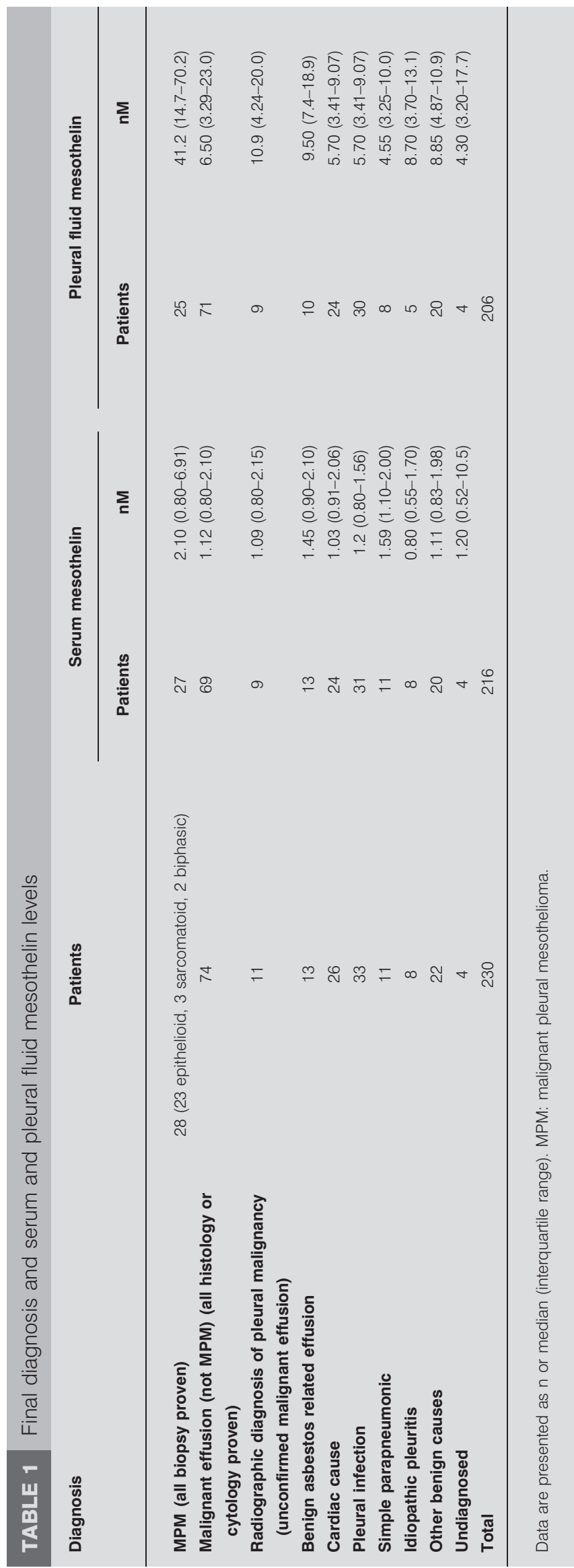



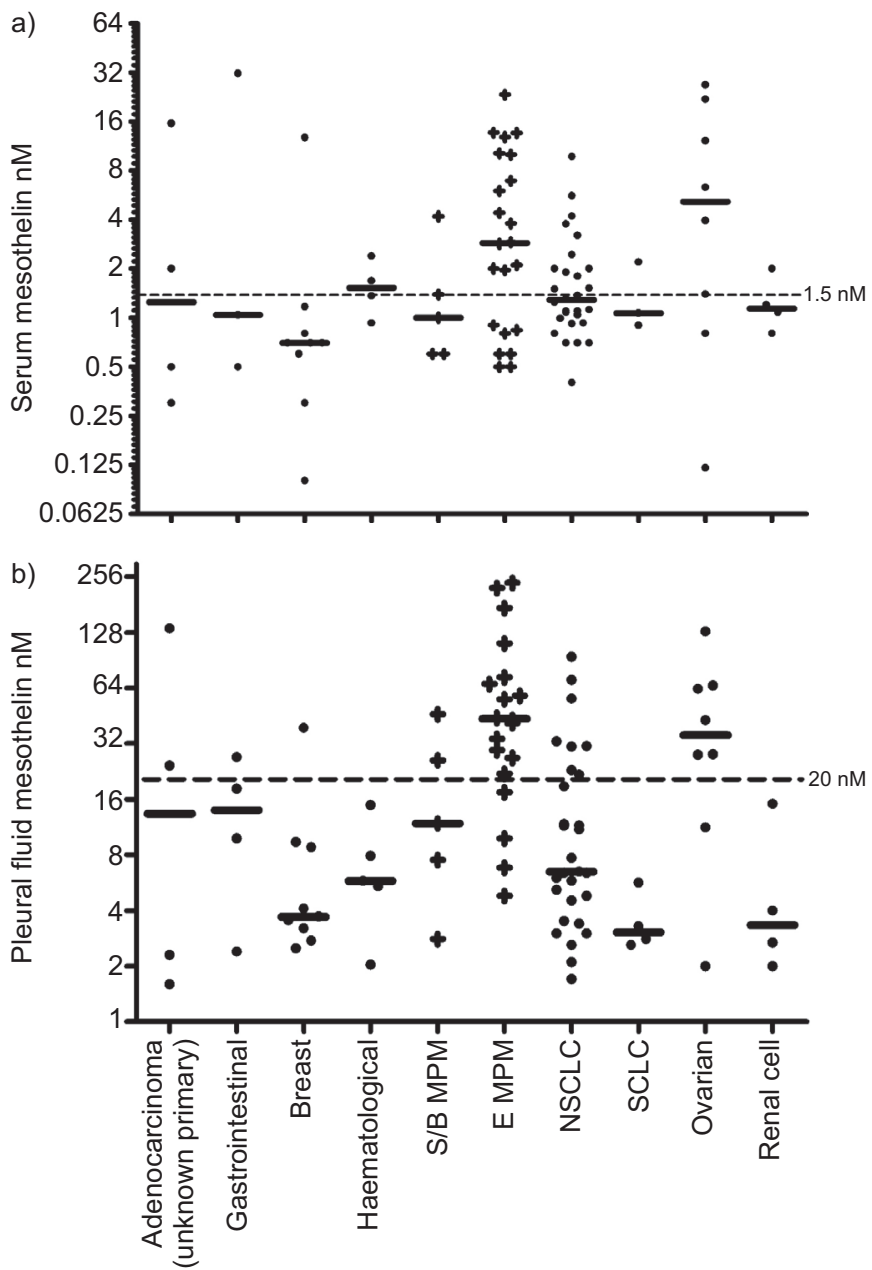

Histological malignant subtype

FIGURE 2. a) Serum and b) pleural fluid mesothelin levels in histological subtypes of patients with malignant pleural effusions. Median levels in each group and positive cut-off level for mesothelin are shown. S/B MPM: sarcomatoid/biphasic malignant pleural mesothelioma; E MPM: epithelioid MPM; NSCLC: nonsmall cell lung cancer; SCLC: small cell lung cancer.

False-negative serum mesothelin was seen in three out of three patients with sarcomatoid MPM, one out of two biphasic MPM patients and seven out of 22 patients with epithelioid MPM, while a false-negative pleural fluid mesothelin was seen in three out of three sarcomatoid MPM and four out of 20 epithelioid MPM cases for whom samples were available.

\section{Diagnostic characteristics of mesothelin for distinguishing MPM from other causes of pleural effusion}

Excluding unconfirmed malignancy (radiographic diagnosis) and undiagnosed patients, serum mesothelin was examined in the group as a whole at a cut-off of $1.5 \mathrm{nM}$, yielding sensitivity $59.3 \%$ (95\% CI $38.8-77.6 \%$ ), specificity $64.7 \%$ (95\% CI $57.2-$ 72.0\%), PPV 20.5\% (95\% CI 12.2-31.1\%) and NPV 91.2\% (95\% CI 84.8-95.5\%) (table 2).

Pleural fluid mesothelin was examined at a cut-off of $20 \mathrm{nM}$, giving sensitivity $72.0 \%$ (95\% CI $50.6-87.9 \%$ ), specificity $87.5 \%$ (95\% CI 81.5-92.8\%), PPV 46.2\% (95\% CI 30.1-62.8\%), NPV 95.5\% (95\% CI 90.8-98.2\%) (table 3).
TABLE 2 Diagnostic characteristics of serum mesothelin at a cut-off of $1.5 \mathrm{nM}$

\begin{tabular}{lccc}
$\begin{array}{l}\text { Serum } \\
\text { mesothelin }\end{array}$ & MPM & Not MPM & Total \\
\hline $\begin{array}{l}\text { 1.5 nM } \\
<1.5 \mathrm{nM}\end{array}$ & 16 & $62^{\#}$ & 78 \\
Total & $11^{\circ}$ & 114 & 125 \\
\hline
\end{tabular}

Data are presented as n. MPM: malignant pleural mesothelioma. ${ }^{\#}$ : falsepositive results occurred in 36 patients with benign pleural effusions and 26 with non-MPM malignant pleural effusions; ": false-negative results were seen in three patients with sarcomatoid, one biphasic and seven epithelioid MPM

\section{Mesothelin testing within the investigation pathway for an undiagnosed pleural effusion}

Data regarding the diagnostic accuracy of mesothelin testing in the context of cytology, CT scan and pleural biopsy results, and in patients with a final diagnosis of BAPE are given in the online supplementary material.

\section{Comparative diagnostic accuracy of mesothelin measured in serum and pleural fluid}

Considering only the 182 patients with a confirmed final diagnosis and both serum and pleural fluid mesothelin results (24 with MPM), the AUC for serum mesothelin in the diagnosis of mesothelioma was 0.61 (95\% CI 0.46-0.77), which is significantly inferior to the AUC for pleural fluid at 0.85 (95\% CI 0.77-0.94; $\mathrm{p}=0.0001$ ) (fig. 3).

Diagnostic cut-off levels derived from both ROC curves at 95\% specificity gave sensitivity $25.0 \%$ (95\% CI $12.0-45.0 \%$ ), PPV $46.0 \%(95 \%$ CI $23.0-71.0 \%)$ and NPV $89.0 \%$ (95\% CI $84.0-$ $93.0 \%$ ) for serum (cut-off $0.975 \mathrm{nM}$ ), and sensitivity $50.0 \%(95 \%$ CI $31.0-69.0 \%)$, PPV $63.0 \%$ (95\% CI $41.0-81.0 \%)$ and NPV 93.0\% (95\% CI 87.0-96.0\%) for pleural fluid (cut-off $43.0 \mathrm{nM}$ ).

\section{The impact of significant CKD}

Contemporaneous creatinine and eGFR measurements were available for 229 out of 230 patients at the time of sample collection.

$74(32 \%)$ out of 229 patients had an eGFR $\leqslant 59 \mathrm{~mL} \cdot \mathrm{min}^{-1}$ (stage 3 CKD or greater).

\section{TABLE 3 Diagnostic characteristics of pleural fluid mesothelin at a cut-off of $20 \mathrm{nM}$}

\begin{tabular}{|c|c|c|c|}
\hline $\begin{array}{l}\text { Pleural fluid } \\
\text { mesothelin }\end{array}$ & MPM & Not MPM & Total \\
\hline$\geqslant 20.0 \mathrm{nM}$ & 18 & $21^{\#}$ & 39 \\
\hline$<20.0 \mathrm{nM}$ & $7^{\bullet}$ & 147 & 154 \\
\hline Total & 25 & 168 & 193 \\
\hline
\end{tabular}

Data are presented as n. MPM: malignant pleural mesothelioma. \#: False positive results occurred in two patients with benign pleural effusions and 19 with non-MPM malignant pleural effusions; ${ }^{\bullet}$ : false-negative results were seen in three patients with sarcomatoid MPM and four patients with epithelioid MPM. 


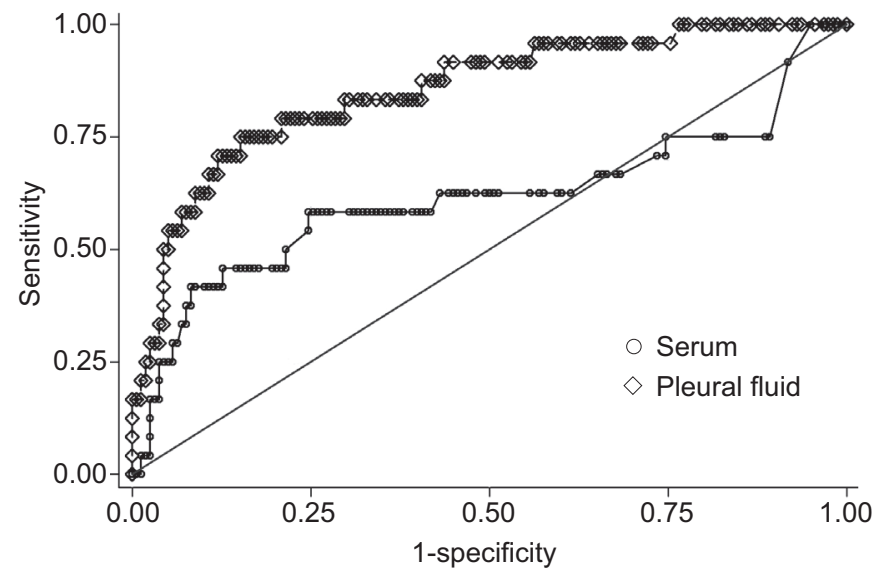

FIGURE 3. Receiver operating characteristics curves comparing the diagnostic accuracy of serum and pleural fluid mesothelin testing for the diagnosis of malignant pleural mesothelioma. Patients with both sample types and a definitive final diagnosis were included $(n=182)$.

Specificity of serum mesothelin was 72.3\% (95\% CI 63.3-80.1\%) in patients with an eGFR $>59 \mathrm{~mL} \cdot \mathrm{min}^{-1}$ but $49.1 \%(95 \%$ CI $35.6-$ $62.7 \%$ ) in those with eGFR $\leqslant 59 \mathrm{~mL} \cdot \mathrm{min}^{-1}$ (tables 4 and 5). $19(54 \%)$ out of 35 patients with a benign cause for pleural effusion but eGFR $\leqslant 59 \mathrm{~mL} \cdot \mathrm{min}^{-1}$ had a false-positive serum mesothelin result.

Diagnostic characteristics of pleural fluid mesothelin were unaffected by renal function. Specificity with eGFR $>59 \mathrm{~mL} \cdot \mathrm{min}^{-1}$ was $86.0 \%(95 \%$ CI $78.2-91.8 \%)$ and with eGFR $\leqslant 59 \mathrm{~mL} \cdot \mathrm{min}^{-1}, 87.0 \%$ (95\% CI 75.1-94.6\%).

In patients with a confirmed non-MPM diagnosis, median serum mesothelin was $1.54(1.00-2.40) \mathrm{nM}$ in patients with eGFR $\leqslant 59 \mathrm{~mL} \cdot \mathrm{min}^{-1}$, significantly higher than with an eGFR $>59 \mathrm{~mL} \cdot \mathrm{min}^{-1}$ at $1.1(0.80-1.59) \mathrm{nM}$. Median pleural fluid mesothelin was not significantly different with (6.22 (3.0$11.07) \mathrm{nM}$ ) and without (5.41 (2.66-10.99) $\mathrm{nM})$ renal impairment (fig. 4).

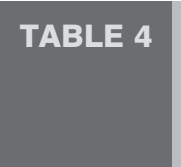

Diagnostic accuracy of serum mesothelin in patients with estimated glomerular filtration rate $>59 \mathrm{~mL} \cdot \mathrm{min}^{-1}$, excluding those undiagnosed or with unconfirmed malignancy

\begin{tabular}{lll}
$\begin{array}{l}\text { Serum } \\
\text { mesothelin }\end{array}$ & MPM & Not MPM \\
\hline
\end{tabular}

\begin{tabular}{lccc}
\hline$\geqslant 1.5 \mathbf{n M}$ & 9 & 33 & 42 \\
$<1.5 \mathbf{n M}$ & 10 & 86 & 96 \\
Total & 19 & 141 & 138 \\
Sensitivity \% & & $47.4(24.5-71.2)$ & \\
Specificity \% & & $72.3(63.3-80.1)$ & \\
PPV \% & & $21.0(10.3-36.8)$ & \\
NPV \% & & $89.6(81.6-94.9)$ & \\
\hline
\end{tabular}

Data are presented as $\mathrm{n}$ or $\%(95 \% \mathrm{Cl})$. MPM: malignant pleural mesothelioma; PPV: positive predictive value; NPV: negative predictive value.

\begin{tabular}{|c|c|c|c|}
\hline TABLE 5 & \multicolumn{3}{|c|}{$\begin{array}{l}\text { Diagnostic accuracy of serum mesothelin in } \\
\text { patients with estimated glomerular filtration rate } \\
\leqslant 59 \mathrm{~mL} \cdot \mathrm{min}^{-1} \text { (chronic kidney disease stage } \\
\geqslant 3 \text { ), excluding those undiagnosed or with } \\
\text { unconfirmed malignancy }\end{array}$} \\
\hline $\begin{array}{l}\text { Serum } \\
\text { mesothelin }\end{array}$ & MPM & Not MPM & Total \\
\hline$\geqslant 1.5 \mathrm{nM}$ & 7 & 29 & 36 \\
\hline$<1.5 \mathrm{nM}$ & 1 & 28 & 29 \\
\hline Total & 8 & 57 & 65 \\
\hline Sensitivity \% & & $88.9(51.8-99.7$ & \\
\hline Specificity \% & & $49.1(35.6-62.7$ & \\
\hline PPV \% & & $21.6(9.8-38.2$ & \\
\hline NPV \% & & $96.6(82.2-99.9$ & \\
\hline
\end{tabular}

Data are presented as n or \% $(95 \% \mathrm{Cl})$. MPM: malignant pleural mesothelioma; PPV: positive predictive value; NPV: negative predictive value.

There was no statistically significant association between age and serum or pleural fluid mesothelin when adjustment was made for the association between falling eGFR and increasing age.

\section{DISCUSSION}

This study has examined the practical application of mesothelin testing in serum and pleural fluid, in consecutive patients presenting with an undiagnosed pleural effusion.

Consistent with previous investigators, we have demonstrated significantly higher levels of mesothelin in serum and pleural fluid in patients with histologically confirmed mesothelioma than with pleural effusions of another cause, but a clinically important rate of false-positive results in patients with a wide range of non-MPM malignant sub-types as well as benign disease [8, 9, 13-17].

Mesothelin is expressed on normal mesothelium [18] and gene expression studies confirm overexpression on malignant cells of many histological sub-types including MPM, ovarian, pancreatic, endometrial, lung, oesophageal, colonic, vulval and cervical malignancies [19]. Lack of specificity amongst non-MPM malignant pleural effusions is therefore explicable and well documented, but does represent a substantial limitation to the clinical utility of the test.

False-positive serum results in patients with benign diagnoses were also very common in our series; 36 (34\%) out of 107 patients with a benign diagnosis had a positive serum mesothelin at a cut-off level of $1.5 \mathrm{nM}$. Pleural fluid mesothelin was positive in only two patients with benign disease; both had a final diagnosis of robustly ascertained BAPE.

Positive results in patients with BAPE were also seen commonly with the serum test (five out of 13 patients). All patients had a lack of radiographic progression on serial CT scan for at least 12 months and all but one had irrefutable histological confirmation of benign disease.

Immuno-histochemical studies have shown that most sarcomatoid sub-type MPM tissue does not express mesothelin, such that the "false" negative test results shown in this and 

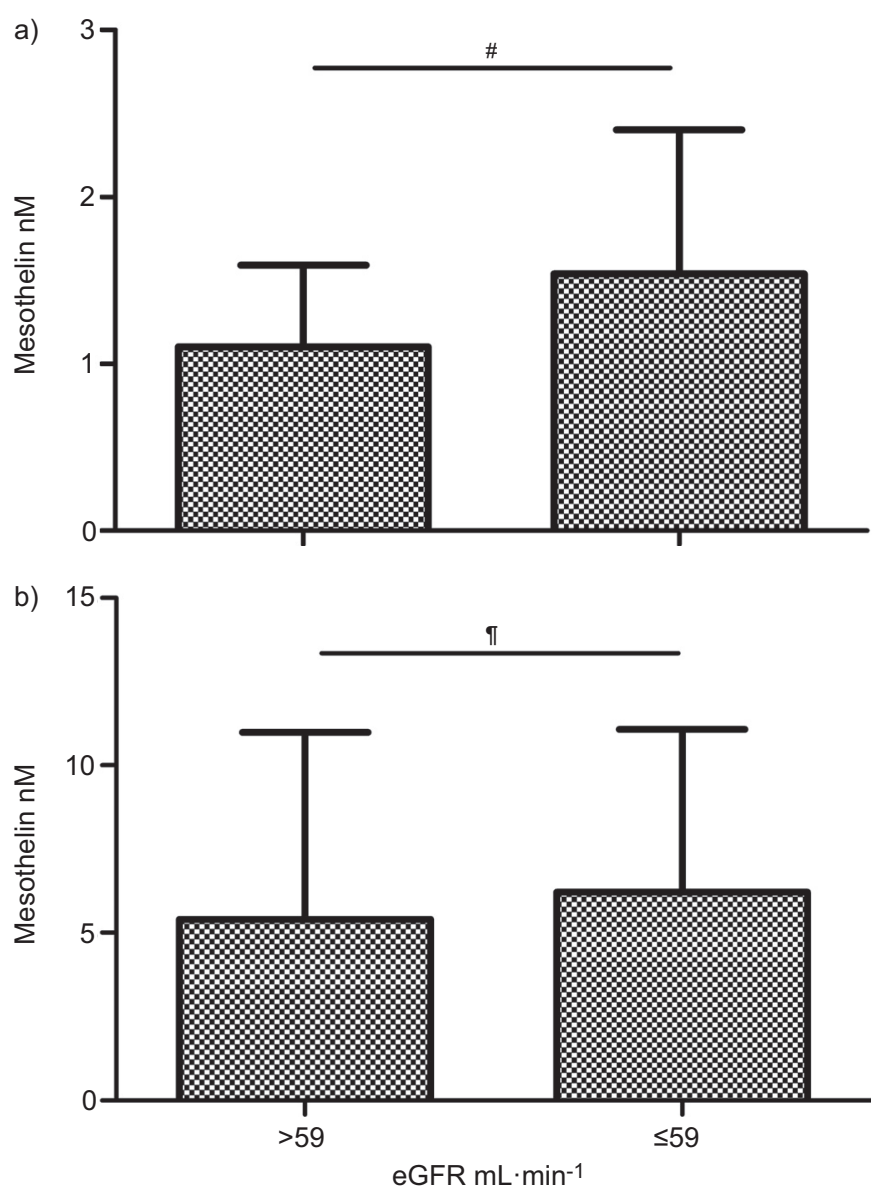

FIGURE 4. a) Serum and b) pleural fluid mesothelin in patients with a nonmalignant pleural mesothelioma diagnosis with (estimated glomerular filtration rate (eGFR) $\leqslant 59 \mathrm{~mL} \cdot \mathrm{min}^{-1}$ ) and without (eGFR $>59 \mathrm{~mL} \cdot \mathrm{min}^{-1}$ ) significant renal failure Data are presented as median and interquartile range. ${ }^{\#}: p=0.007 ;{ }^{\circ}: p=0.605$.

previous studies are to be expected [20]. Serum and pleural fluid mesothelin were also negative in $32 \%$ and $20 \%$ of patients with epithelioid sub-type disease, presumably reflecting variable gene expression.

We have demonstrated superior diagnostic characteristics for pleural fluid mesothelin above serum within paired results alone, at a fixed specificity of $95 \%$, and using established cut-off levels, at each stage in the diagnostic pathway. This contrasts with the conclusion of several earlier series examining smaller numbers of paired results that have not demonstrated a significant difference between pleural fluid and serum analysis [8, 13].

Overall the NPV of mesothelin when measured in serum (91.2\%) and pleural fluid (95.5\%) is good, but the PPV $(20.5 \%$ and $46.2 \%$, respectively) is not within a clinically useful range.

A recent study by BoudviLLE et al. [10] examined serum mesothelin levels in 144 patients with stable CKD but no clinically apparent pleural disease and demonstrated significantly higher levels in patients with CKD stage 3 or greater compared to those with CKD stage 2, suggesting that mesothelin, released from normal mesothelial cells, undergoes a degree of renal clearance, consistent with its small molecular mass. HolleVoet et al. [11] measured serum mesothelin and glomerular filtration rate (GFR) in 66 control patients, demonstrating a positive correlation between GFR and the reciprocal of serum mesothelin and a false-positive rate of $52 \%$ in patients with CKD stage 3 or greater.

In our series, specificity of serum mesothelin was $72.3 \%$ in patients with an eGFR $>59 \mathrm{~mL} \cdot \mathrm{min}^{-1}$, compared with $49.1 \%$ in patients with CKD stage 3 or greater. 20 out of 36 positive serum tests in patients with benign pleural effusions may be explained by significant renal failure. Levels of mesothelin measurable in pleural fluid in patients without mesothelioma were not affected by renal function and the diagnostic accuracy of the test when applied to pleural fluid was not significantly different between the two renal function groups. While accumulation of mesothelin in pleural fluid as a result of diminishing renal clearance would not necessarily be expected (as it would in serum), this is the first study to demonstrate this potential advantage of pleural fluid testing.

There are several notable limitations to this study. While it is one of the largest reported series of patients presenting with pleural effusions to undergo mesothelin testing, the proportion of patients with mesothelioma was low when compared with most previous studies that have selected samples from established tissue banks by diagnosis. However, this proportion accurately reflects our consecutive patient population. We studied the diagnostic accuracy of mesothelin at previously established cut-off levels, which, while achieving the study aim (to examine the test in a clinically relevant manner), may produce inferior diagnostic characteristics than derivation of cut-off levels specific to our patient population or according to a desired sensitivity or specificity. Finally, conclusions regarding the utility of the test when applied to pleural fluid are tempered by the current lack of validation of commercially available ELISAs for this sample type.

The strength of this study is the recruitment of an unselected consecutive series of patients with pleural effusions requiring investigation and the robust application of diagnostic criteria, diagnosis of MPM exclusively on histological grounds and patient follow-up to 12 months in the presence of benign pleural disease. Within this complex "real world" sample, the specificity and PPV of mesothelin are challenged.

In conclusion, these results suggest that the routine testing of mesothelin in all undiagnosed pleural effusions is unhelpful. If the test is performed for diagnostic purposes, pleural fluid should be used instead of serum, particularly in patients with an eGFR $\leqslant 59 \mathrm{~mL} \cdot \mathrm{min}^{-1}$. A negative result in a patient with a low pre-test probability of MPM is reassuring for an absence of epithelioid mesothelioma and NPV appears to be relatively consistent between serum and pleural fluid. Positive test results, however, should be interpreted with caution, as false positives are common, including in patients with BAPE. The role of the test in MPM diagnosis therefore appears limited. Serum mesothelin has shown promise in monitoring treatment response and disease progression [21-24] and this should be the focus of future studies.

\section{CLINICAL TRIAL}

This study is registered at UK Clinical Research Network with adoption number 8960 . 


\section{STATEMENT OF INTEREST}

Statements of interest for J.E. Harvey and N.A. Maskell, and for the study itself can be found at www.erj.ersjournals.com/site/misc/ statements.xhtml

\section{ACKNOWLEDGEMENTS}

The authors would like to acknowledge M. Darby and I. Laurence (Dept of Radiology, North Bristol NHS Trust, Bristol, UK) for their assistance with radiological aspects of the study, N. Ibrahim and M. Brett (Dept of Histopathology, North Bristol NHS Trust) for their histology and cytology contributions and K. Elvers (University of Bristol, Bristol) for assisting with mesothelin assays. Mesomark ${ }_{\circledR}$ test kits were provided at reduced cost by Fujirebio Diagnostics Inc. (Malvern, PA, USA).

\section{REFERENCES}

1 Musk AW, De Klerk N. Epidemiology of malignant mesothelioma in Australia. Lung Cancer 2004; 45: S21-S23.

2 Light RW, Lee YCG, eds. Textbook of Pleural Diseases. 2nd Edn. London, Hodder Arnold, 2008.

3 British Thoracic Society Standards of Care Committee. BTS statement on malignant mesothelioma in the UK, 2007. Thorax 2007; 62: Suppl. 2, ii1-ii19.

4 Kutty CPK, Remeniuk E, Varkey B. Malignant-appearing cells in pleural effusions due to pancreatitis. Acta Cytol 1981; 25: 412-416.

5 Traill ZC, Davies RJO, Gleeson FV. Thoracic computed tomography in patients with suspected malignant effusions. Clin Radiol 2001; 56: 193-196.

6 Hooper CE, Lee YCG, Maskell NA. Investigation of a unilateral pleural effusion in adults: British Thoracic Society pleural disease guideline 2010. Thorax 2010; 65: Suppl. 2, ii4-ii17.

7 Luo L, Shi H-Z, Liang Q-L, et al. Diagnostic value of soluble mesothelin-related peptides for malignant mesothelioma: a metaanalysis. Respir Med 2010; 104: 149-156.

8 Creaney J, Yeoman D, Naumoff LK, et al. Soluble mesothelin in effusions: a useful tool for the diagnosis of malignant mesothelioma. Thorax 2007; 62: 569-576.

9 Davies HE, Sadler RS, Bielsa S, et al. Clinical Impact and reliability of pleural fluid mesothelin in undiagnosed pleural effusions. Am J Respir Crit Care Med 2009; 180: 437-444.
10 Boudville N, Paul R, Robinson BWS, et al. Mesothelin and kidney function - analysis of relationship and implications for mesothelioma screening. Lung Cancer 2011; 73: 320-324.

11 Hollevoet K, Bernard D, De Geeter F, et al. Glomerular filtration rate is a confounder for the measurement of soluble mesothelin in serum. Clin Chem 2009; 55: 1431-1433.

12 Hollevoet K, Nackaerts K, Thas O, et al. The effect of clinical covariates on the diagnostic and prognostic value of soluble mesothelin and megakaryocyte potentiating factor. Chest 2012; 141: 477-484.

13 Scherpereel A, Grigoriu B, Conti M, et al. Soluble mesothelinrelated peptides in the diagnosis of malignant pleural mesothelioma. Am J Respir Crit Care Med 2006; 173: 1155-1160.

14 Creaney J, Van Bruggen I, Hof M, et al. Combined CA125 and mesothelin levels for the diagnosis of malignant mesothelioma. Chest 2007; 132: 1239-1246.

15 Cristaudo A, Foddis R, Vivaldi A, et al. Clinical significance of serum mesothelin in patients with mesothelioma and lung cancer. Clin Cancer Res 2007; 13: 5076-5081.

16 Robinson BW, Creaney J, Lake R, et al. Mesothelin-family proteins and diagnosis of mesothelioma. Lancet 2003; 362: 1612-1616.

17 Creaney J, Christansen H, Lake R, et al. Soluble mesothelin related protein in mesothelioma. J Thorac Oncol 2006; 1: 172-174.

18 Chang K, Pastan I, Willingham MC. Isolation and characterisation of monoclonal antibody $\mathrm{K} 1$, reactive with ovarian cancers and normal mesothelium. Int J Cancer 1992; 50: 373-381.

19 Creaney J, Robinson BW. Detection of malignant mesothelioma in asbestos exposed individuals: the potential role of soluble mesothelinrelated protein. Haematol Oncol Clin North Am 2005; 19: 1025-1040.

20 Ordonez NG. Value of mesothelin immunostaining in the diagnosis of mesothelioma. Mod Pathol 2003; 16: 192-197.

21 Creaney J, Francis RJ, Dick IM, et al. Serum soluble mesothelin concentrations in malignant pleural mesothelioma: relationship to tumour volume, clinical stage and changes in tumour burden. Clin Cancer Res 2011; 17: 1181-1189.

22 Grigoriu BD, Chahine B, Vachani A, et al. Kinetics of soluble mesothelin in patients with malignant pleural mesothelioma during treatment. Am J Respir Crit Care Med 2008; 179: 950-954.

23 Wheatley-Price P, Yang B, Patsios D, et al. Soluble mesothelinrelated peptide and osteopontin as markers of response in malignant mesothelioma. J Clin Oncol 2010; 28: 3316-3322.

24 Hollevoet K, Nackaerts K, Gosselin R, et al. Soluble mesothelin, megakaryocyte potentiating factor, and osteopontin as markers of patient response and outcome in mesothelioma. J Clin Oncol 2011; 6: 1930-1937. 